\title{
The Structure of the Lamina Cribrosa of the Human Eye: an Immunocytochemical and Electron Microscopical Study
}

\author{
A. R. ELKINGTON, C. B. E. INMAN, P. V. STEART, R. O. WELLER \\ Southampton
}

\begin{abstract}
Summary
The structure of the lamina cribrosa was studied by histological and immunocytochemical techniques and by scanning and transmission electron microscopy in four eyes removed surgically and in 21 normal eyes obtained from an eye bank. Dissection of the eyes emphasised the relatively fragile links between lamina and sclera. Reticulin staining of the fibrous trabeculae in the posterior (scleral) part of the lamina revealed a structure composed of interweaving skeins of collagen fibres frequently arranged tangentially around the canals, 40-220 $\mu \mathrm{m}$ in diameter, through which optic nerve axons pass. Immunocytochemistry for glial fibrillary acidic protein demonstrated the intimate web that astrocyte processes form around axons within the canals of the lamina and the close association of astrocyte processes and fibrous trabeculae in the posterior part of the lamina. Scanning electron microscopy clearly demonstrated anatomical relationships of the lamina cribrosa and the wide variation in the size of the canals in the lamina. Transmission electron microscopy confirmed the close association of astrocyte processes with axons demonstrated in the immunocytochemical preparations. The results of this study emphasise the complex relationships between astrocytic, neural and fibrous elements in the lamina cribrosa and how more information is required regarding the mechanical and metabolic properties of the astrocyte web and the fibrous trabeculae before the role of the lamina cribrosa in the pathogenesis of nerve damage in glaucoma can be fully assessed.
\end{abstract}

Interest in the structure of the lamina cribrosa of the human eye has been aroused particularly through the detection of axon balloons, and thus axon damage, in the optic nerve at the lamina cribrosa both in glaucoma ${ }^{1-3}$ and in papilloedema. ${ }^{4,5}$ It has been suggested that either vascular factors ${ }^{6}$ or the structure of the lamina cribrosa itself ${ }^{3,7,8}$ could account for the damage to optic nerve fibres at this site, but the exact mechanisms for axonal damage have not been established. Previous histological ${ }^{3,8}$ and ultrastructural ${ }^{9}$ studies of the lamina have emphasised its fibrous nature and astrocyte content. However, the precise relationships of the astrocytic and fibrous components to the non-myelinated axons passing through the lamina have not been established in such a way that allows direct comparison between normal and pathological eyes.

The objectives of the present study are to investigate the relationships between astrocytic, fibrous and neural components in the 

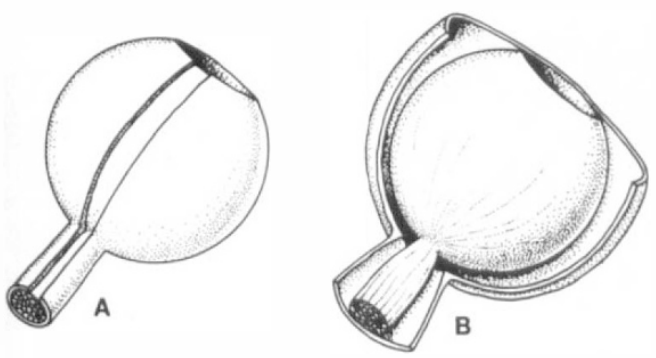

Fig. 1. Dissection of the eye for transmission microscopy. Diagram to show how incisions were made through both the dural sheath of the optic nerve and the sclera $(A)$. The dura and sclera were peeled off the underlying nerve and the retina which could then be removed in continuity $(B)$.

lamina cribrosa using histological and immunohistochemical techniques. These observations will be correlated with the structure of the lamina cribrosa observed by scanning and transmission electron microscopy.

\section{Material and Methods}

Twenty-one eyes from patients aged 17-45 years were donated by the Bristol Eye Bank. Following removal of the cornea, the eyes were fixed in buffered formalin at $\mathrm{pH} 7.2$ for at least 24 hours. Four eyes were surgically removed from patients 50-65 years old with malignant melanomas of the choroid not affecting the optic disc areas. Immediately after removal, the eye was opened by a coronal incision and the posterior part of the eye fixed in $5 \%$ glutaraldehyde in $0.1 \mathrm{M}$ cacodylate buffer at $\mathrm{pH} 7.2$ for 24 hours; these nerves were used for transmission electron microscopy. The optic discs of all eyes used were inspected under a dissecting microscope and were found to be macroscopically normal.

\section{Light microscopy}

Formalin fixed eyes were embedded in paraffin wax and sagittal (longitudinal) or transverse sections $6 \mu \mathrm{m}$ cut through the optic disc, lamina cribrosa and proximal part of the optic nerve. Adjacent sections were stained with haematoxylin and eosin, haematoxylin van Giesen, and the Gordon and Sweet technique for reticulin.

For the immunocytochemical demonstration of glial fibrillary acidic protein (GFAP) alone, the standard ABC immuno- peroxidase technique ${ }^{10}$ was used. Sections were incubated with anti-GFAP serum raised in rabbits from GFAP prepared from a cerebellar astrocytoma by the method of Palfreyman et al. ${ }^{11}$ and diluted 1:2000.

A double-labelling technique was used to localise GFAP and neurofilament protein in the same section using rabbit anti-GFAP polyclonal antibody and a mouse monoclonal antibody to the $70 \mathrm{kd}$ neurofilament protein (Tissue Culture Services). Peroxidase was developed as a brown colour to locate the neurofilament protein, and alkaline phosphatase $^{12}$ reacted with naphthol ASB 1- fastred-TR was used to localise the GFAP as a pink-red colour.

\section{Scanning Electron Microscopy (SEM)}

Optic nerves fixed in neutral buffered formalin were washed overnight in $0.1 \mathrm{M}$ cacodylate buffer at $\mathrm{pH} 7.2$ and post-fixed in $2 \%$ osmium tetroxide for two hours. Following fixation, some specimens were soaked in the cryoprotectant dimethylsulphoxide (DMSO), frozen, and fractured with a razor blade in order to reveal fresh surfaces for SEM. Three optic discs were embedded in PEG wax using the method of Nagele et al. ${ }^{13}$ and the lamina exposed for SEM. All specimens were dehydrated through graded ethanols and critical point dried, mounted on stubs, and sputter coated and examined in a Hitachi S800 field emission scanning electron microscope.

\section{Transmission Electron Microscopy (TEM)}

Specimens for TEM were fixed immediately in $5 \%$ glutaraldehyde in $0.1 \mathrm{M}$ cacodylate buffer for 24 hours at $4^{\circ} \mathrm{C}$. Following an overnight wash in sucrose-cacodylate buffer, specimens were dissected by incising the dural sheath so that the incision joined up with the corresponding incision made through the sclera (Fig. 1). It was then possible to peel the scleral-dural envelope from the underlying retina and optic nerve. Thus the retina and optic nerve could be removed in continuity with a minimum of further dissection. The optic disc, lamina cribrosa and distal portion of the optic nerve were excised and post-fixed in $2 \%$ osmium tetroxide for two to three hours, stained in the block with $1.5 \%$ aqueous uranyl acetate, dehydrated through graded 


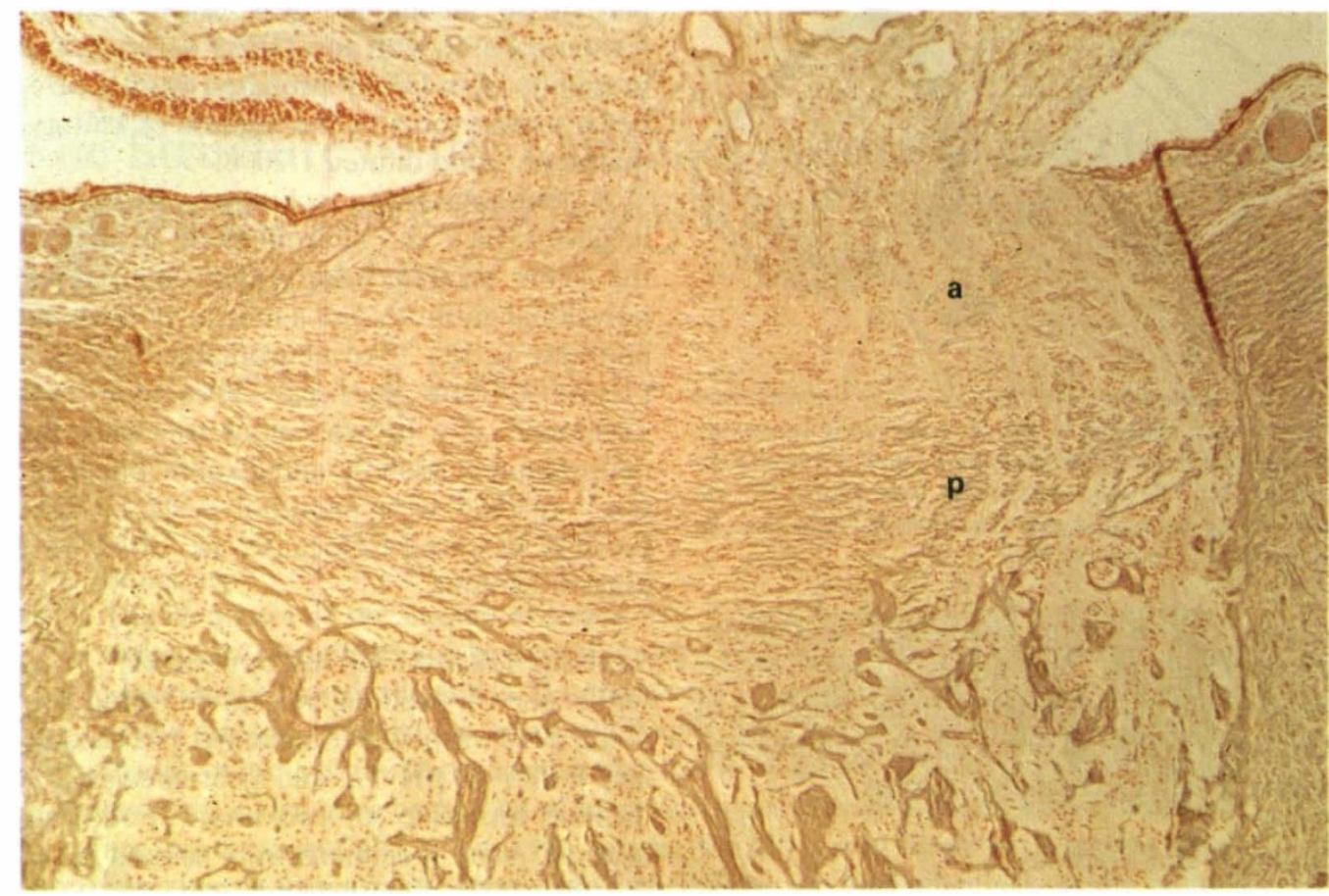

Fig. 2. Sagittal section through the normal lamina cribrosa. Fine collagen fibres are stained black and although prominent in the posterior scleral portion of the lamina $(p)$ collagen is scanty in the anterior (choroidal) portion (a). Gordon and Sweet reticulin stain. $\times 80$.

ethanols and embedded in Spurr's resin, 0.5 $\mu \mathrm{m}$ sections were cut at various levels through the lamina cribrosa, stained with toluidine blue and examined by light microscopy. Thin sections $(17 \mathrm{~nm})$ were cut from selected areas at each of 15 levels through the lamina cribrosa counterstained with Reynold's lead citrate and examined in a Hitachi H-7000 transmission electron microscope.

\section{Results}

Histology and Immunocytochemistry

Histological examination of the laminae cribrosae in this study showed no pathological abnormality or autolytic change.

Sagittal sections through the optic nerve head showed the two major portions of the lamina cribrosa (Figs. 2 and 3). Using a reticulin technique which stains type III collagen fibres (Fig. 2) the anterior (choroidal) portion of the lamina was seen to contain little collagen except around blood vessels. The posterior (scleral) portion, however, contains substantial amounts of collagen (Fig. 2). Almost the converse picture is seen in immunocytochemical preparations stained for glial fibrillary acidic protein (GFAP) (Fig. 3) in which the anterior (choroidal) portion of the lamina is rich in astrocyte processes; astrocytes are also prominent in the posterior portion of the lamina, admixed with the collagenous tissue.

The interrelationships of the major elements of the posterior (scleral) portion of the lamina can be observed in serial sections stained by different techniques. The reticulin fibres in the posterior portion of the lamina are arranged in interlacing skeins (Fig. 4) often orientated in a tangential fashion around the canals through which the bundles of axons pass (Fig. 5). Such canals range in diameter from $25-250 \mu \mathrm{m}$ with variation in canal size in all regions of the lamina. Double immunocytochemical staining (Fig. 5) shows the faint pink colour of the GFAP-rich astrocyte processes associated with the unstained 


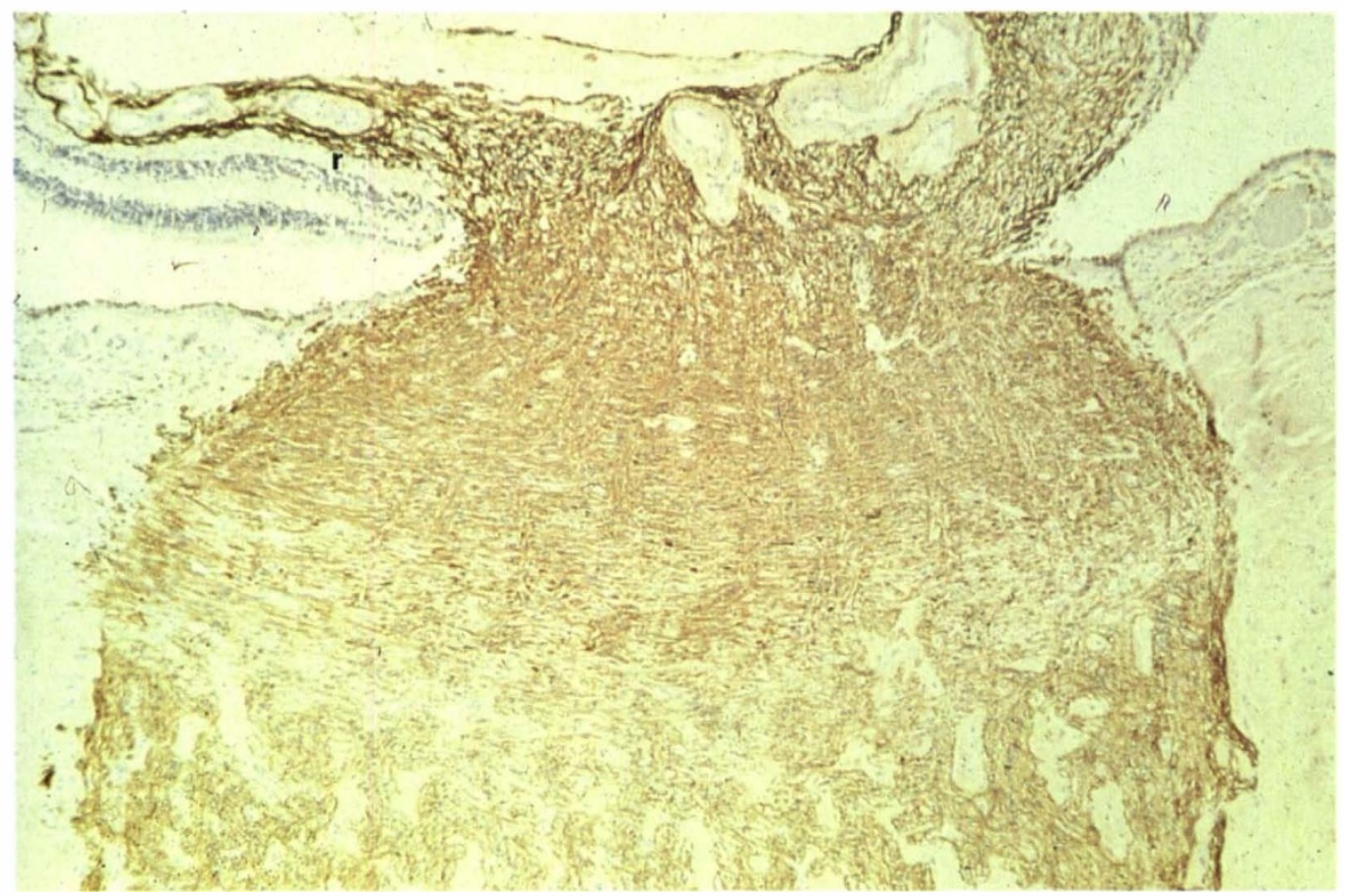

Fig. 3. Sagittal section of lamina cribrosa. Brown astrocyte staining is prominent in the anterior (choroidal) portion of the lamina cribrosa but is less prominent in the posterior (scleral) portion due to the presence of fibrous tissue. Retina $(r)$ is largely unstained. Immunocytochemistry for glial fibrillary acidic protein $(G F A P) . \times 80$.

fibrous trabeculae of the lamina. Bundles of axons passing through the lamina are densely stained for neurofilament protein which obscures the interweaving astrocyte processes. However, the complex interrelationships of the astrocyte processes with both the fibrous trabeculae and the canals through which the nerve fibres pass are seen in Figures 6 and 7. Each canal is divided into many small compartments $2.5-5 \mu \mathrm{m}$ in diameter by a web of fine astrocyte processes. Astrocyte nuclei are arranged around the edges of the canals but in the larger ones, nuclei are also seen in the centre of the canal (Fig. 7). The intimate admixture of astrocyte processes with the fibrous trabeculae is also apparent in Figures 6 and 7; the processes pass over the surface of the collagenous trabeculae and also appear to be embedded within them.

\section{Scanning Electron Microscopy}

A sagittal section through the optic nerve head and the distal portion of the optic nerve
(Fig. 8) reveals the anatomical relationships of the lamina cribrosa. The central retinal vein and artery within the nerve are cut obliquely and, in this view, it is seen how the optic nerve increases in diameter by some $40 \%$ as it leaves the lamina cribrosa. This increase in bulk occurs as the non-myelinated axons in the lamina cribrosa become myelinated in the optic nerve. The subarachnoid space surrounding the optic nerve is seen to extend up to the sclera and the arachnoid trabeculae cross the subarachnoid space to join the pial coating of the optic nerve.

Another view of the posterior (scleral) portion of the lamina cribrosa can be obtained by fracturing the nerve in the transverse plane. In Figure 9a, fractures have occurred at several levels showing the pattern of canals, 25$125 \mu \mathrm{m}$ diameter, in the lamina. At higher power (Fig. 9b), the collagenous trabeculae of the lamina can be seen surrounding canals 35-85 $\mu \mathrm{m}$ in diameter. The axons have become disrupted in this preparation leaving 


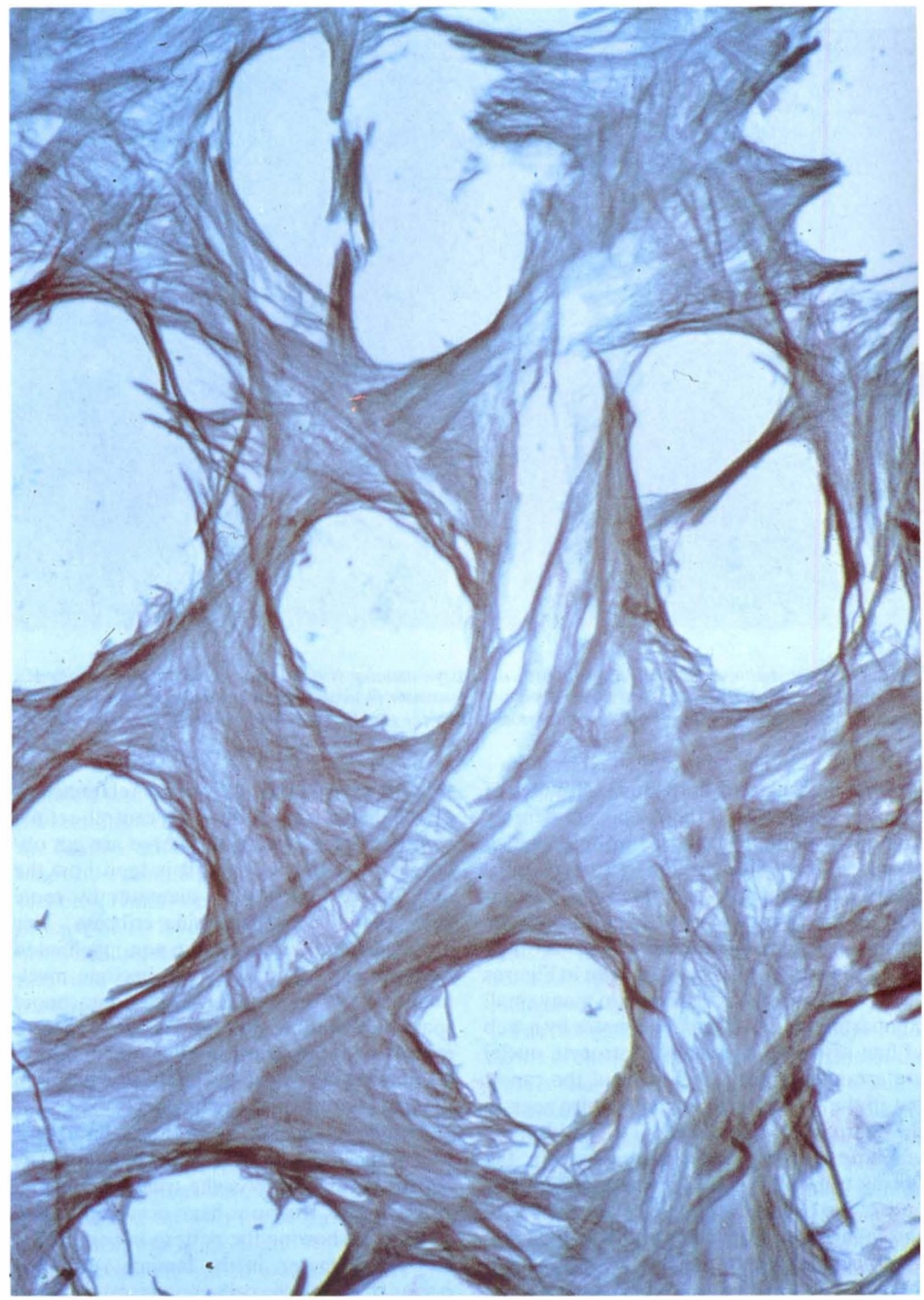

Fig. 4. Transverse section of the scleral lamina cribrosa showing fibrous trabeculae composed of interweaving skeins of fine collagen fibres. The canals through which axons pass range from 40-65 $\mu \mathrm{m}$ and appear to have shelving or rounded edges with collagen bands arranged tangentially. The larger canals are divided by thin collagenous bands. Gordon and Sweet reticulin stain. $\times 715$. 


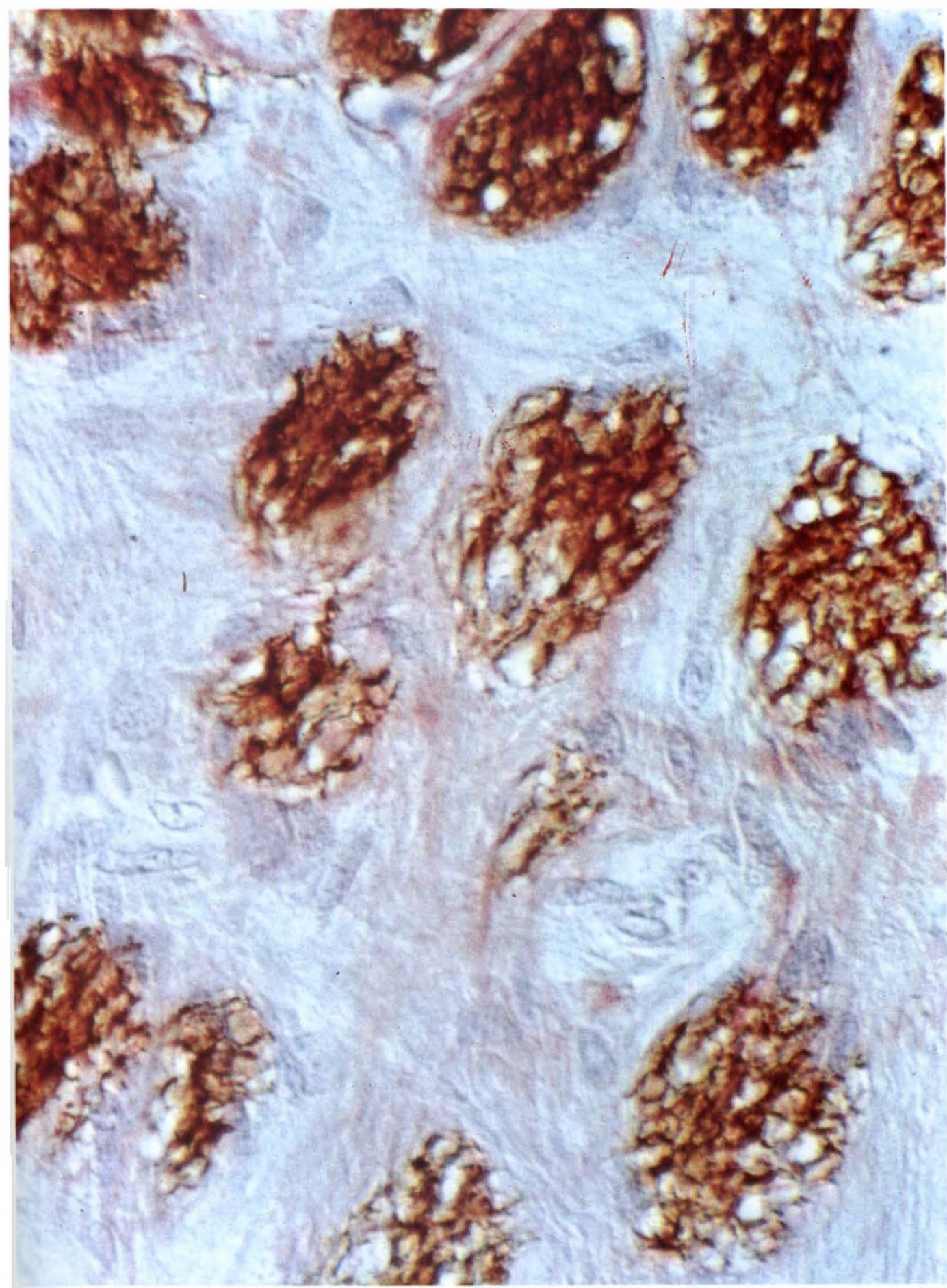

Fig. 5. Groups of axons (brown) in the lamina cribrosa. Astrocyte processes (pink) are closely associated with the unstained collagenous stroma of the lamina. Canals in the lamina, filled with axons, range from 30-60 $\mu \mathrm{m}$ in diameter in this figure. Immunocytochemistry for neurofilament protein (brown-peroxidase) and GFAP (pink). $\times 715$. 


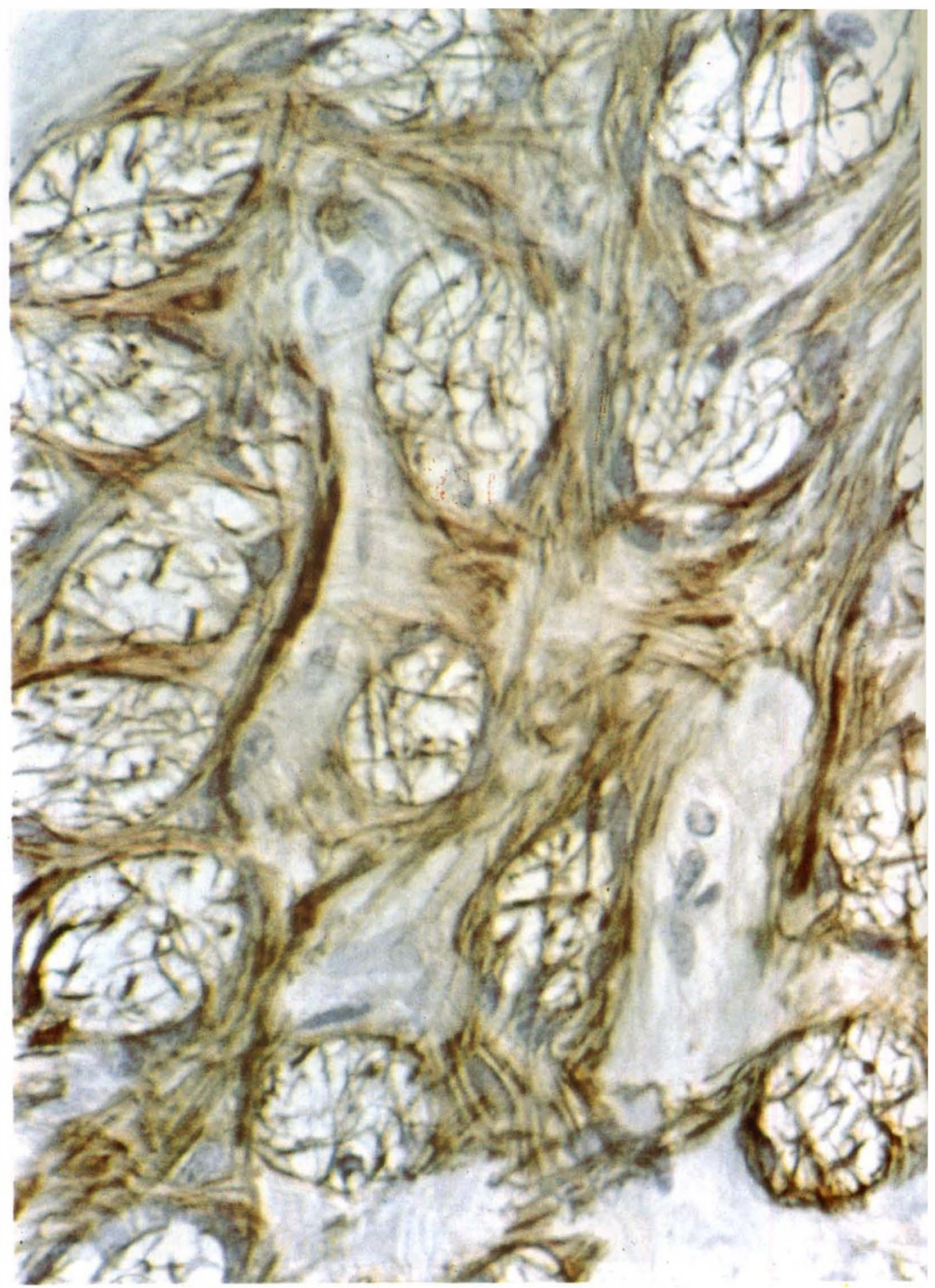

Fig. 6. Similar field to figure 4 stained for astrocytes. Brown filamentous astrocyte processes cover and pierce the unstained collagenous trabeculae. A mesh-like web of astrocyte processes extends across each of the canals in the lamina cribrosa. Immunocytochemistry for GFAP. $\times 715$. 


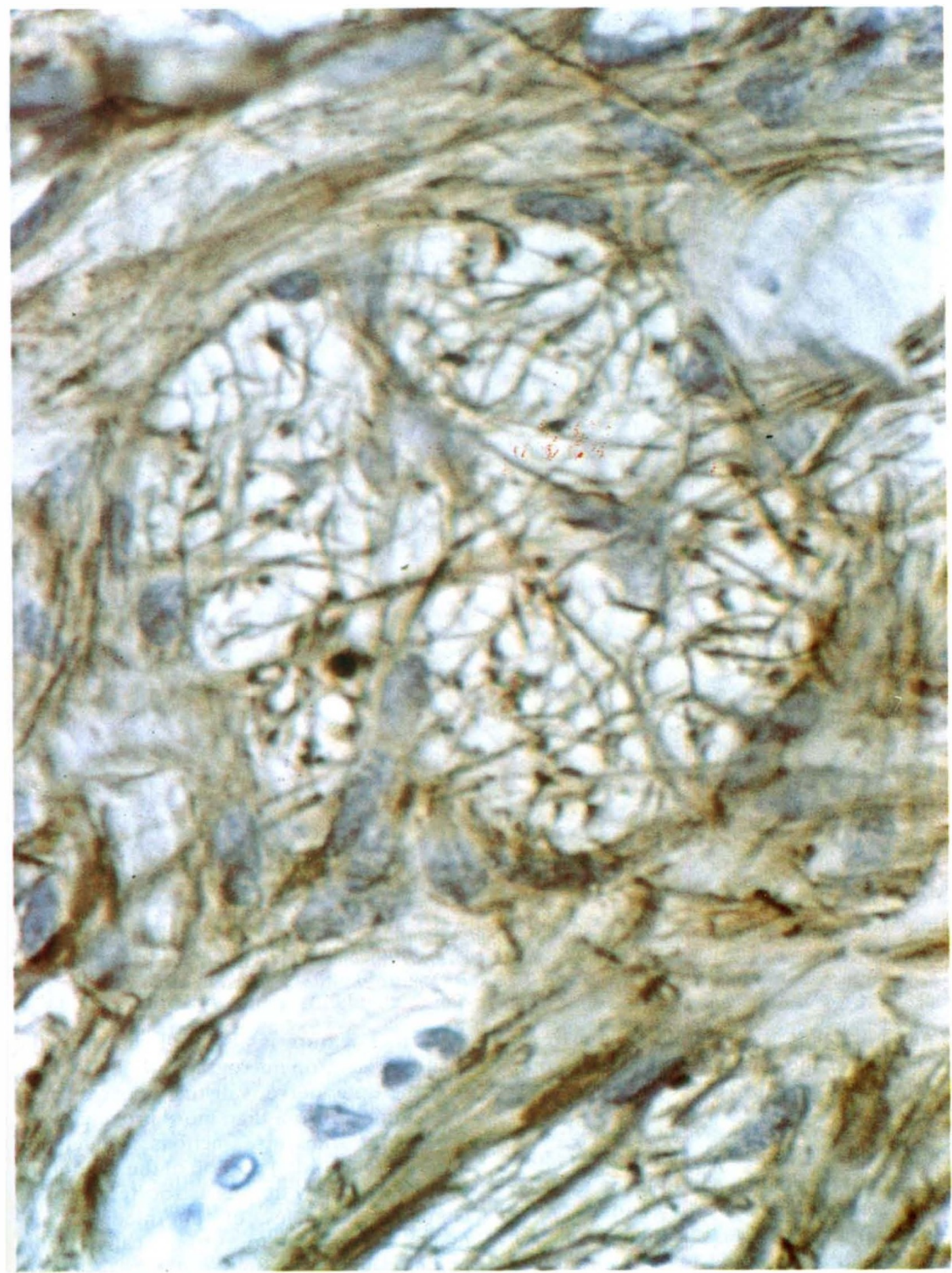

Fig. 7. High power from a preparation similar to Figure 6 showing a web of astrocyte processes spanning a canal in the lamina cribrosa. Astrocyte nuclei (blue) are associated with astrocyte processes (brown) within a canal $85 \mu \mathrm{m}$ in diameter. The perforations in the astrocytic web are 2.5-5.0 $\mathrm{m}$ in diameter. Immunocytochemistry for GFAP. $\times 1100$. 


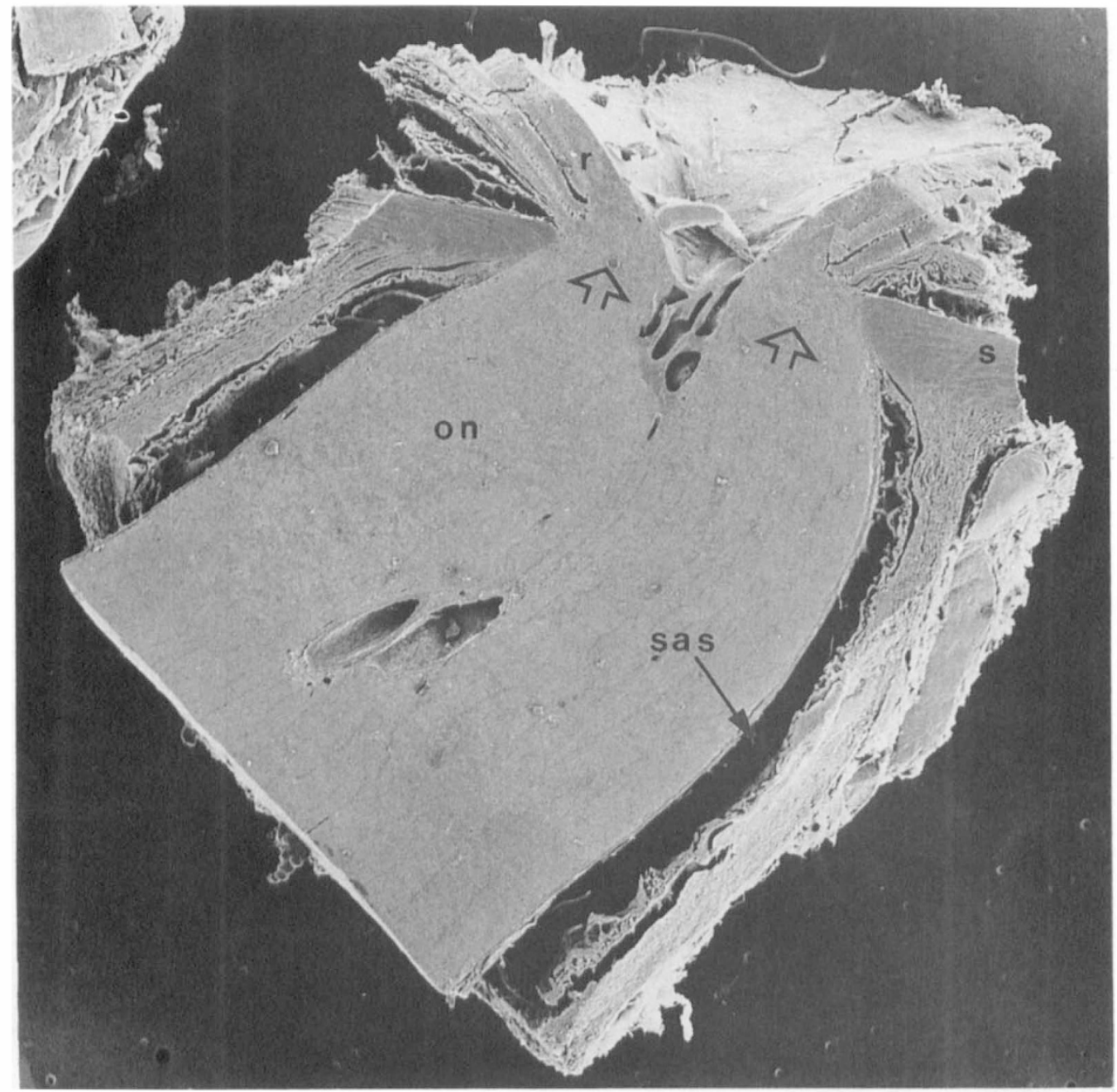

Fig. 8. Scanning electron micrograph (SEM) of sagittal section through the optic nerve (on), lamina cribrosa (open arrows) and part of the retina $(r)$. Central retinal vessels are cut obliquely in the centre of the nerve. Sclera (s). There is an increase in the diameter of the nerve as it leaves the laminal cribrosa. Subarachnoid space (sas) extends up to the sclera. SEM $\times 30$.

a mesh-like pattern resembling the astrocyte web seen in the immunocytochemical preparations (Figs. 6 and 7).

\section{Transmission Electron Microscopy (TEM)}

During the dissection of the optic nerve and lamina cribrosa in preparation for transmission electron microscopy (Fig. 1), it was noted that the optic nerve was only lightly adherent to the sclera and could be easily removed, together with the retina, from the dural and scleral sheath. This suggests that the lamina cribrosa is only lightly attached to the sclera.
A further feature is also well shown in the scanning electron micrograph in Figure 8 i.e. that the optic nerve is distinctly narrower at the lamina cribrosa than more proximally.

TEM of transverse sections through the anterior (choroidal) portion of the lamina cribrosa shows how fibrous astrocyte processes containing bundles of intermediate filaments ${ }^{14}$ form the borders of the canals in the lamina (Fig. 10). Similar fibrous astrocyte processes extend into the canals to form a web and to separate the non-myelinated axons of the optic nerve into bundles of varying size. 


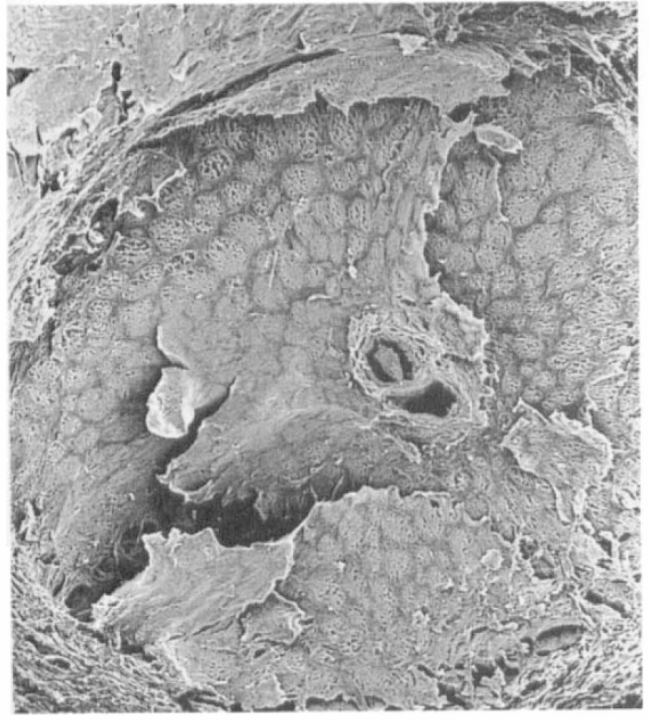

Fig. 9a

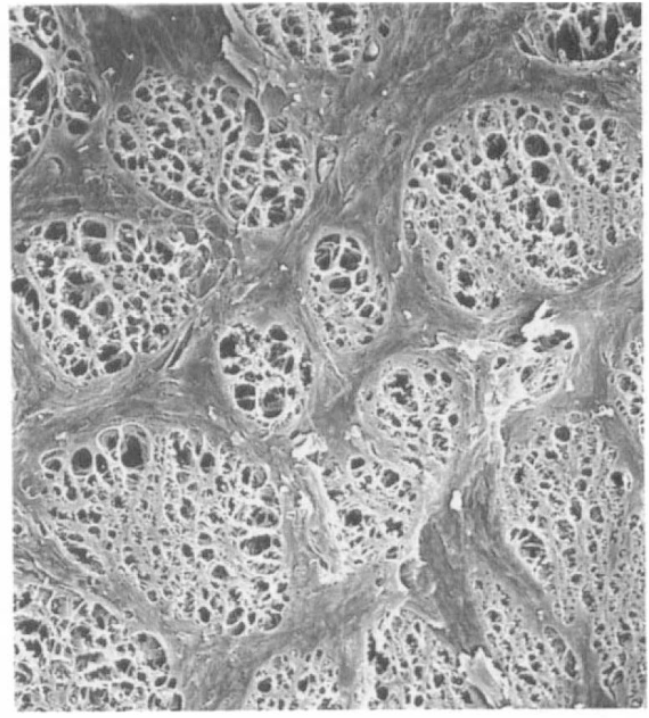

Fig. 9 b

Fig. 9. SEM of scleral lamina cribrosa fractured transversely.

(a) At low magnification, retinal vessels are seen in the centre and the collagenous elements of the lamina cribrosa appear as dark bands; neuroglial elements are pale. Canals in the lamina cribrosa range from 25-125 $\mu \mathrm{m}$.

(b) At higher magnification, the collagenous trabeculae of the lamina cribrosa are seen to surround canals 35-85 $\mu \mathrm{m}$ in diameter filled by a mesh-like pattern suggesting that the axons have swollen post-mortem and that it is mainly the glial elements that are preserved intact.

$a \times 40 b \times 290$.

The astrocyte processes taper and branch as they insinuate themselves between small groups of axons (Fig. 10). Astrocyte nuclei and cell processes are intimately related to some axon bundles (Fig. 11) and a considerable variation in axon diameter, ranging from $0.5-2 \mu \mathrm{m}$, is observed in individual bundles of nerve fibres (Fig. 12). The axons themselves can be identified by their roughly circular cross-sectional profiles and the presence of orientated microtubules and neurofilaments in the cytoplasm. ${ }^{14}$ Intercellular junctional complexes are seen between some of the fibrous astrocyte processes (Fig. 12) and occasional small bundles of collagen fibres are present even within the anterior (choroidal) portion of the lamina cribrosa. As the astrocyte processes taper and insinuate themselves between small groups of axons, the intermediate glial filaments become less prominent (Fig. 12). Despite such intimate contact with astrocytes, many of the axons remain in contact with each other, at least over part of their surface.

In the posterior (scleral) portion of the lamina cribrosa, there is still an intimate mixture of small bundles of axons and astrocyte processes (Fig. 13) but the collagenous elements in the trabeculae are more prominent. Astrocyte processes, surrounded by basement membrane, are embedded within the collagenous trabeculae as suggested by the immunocytochemical preparations. At the edges of the holes in the collagenous lamina, basement membrane is formed where the astrocytes abut on to the collagenous tissue. A similar arrangement of small bundles of axons surrounded by fibrous astrocyte processes is seen within the canals in the trabeculae (Fig. 13). The axon balloon in Figure 13 is the only pathological abnormality observed in the material examined. In the most posterior portion of the collagenous (scleral) lamina, where it is adjacent to the optic nerve, darkly 


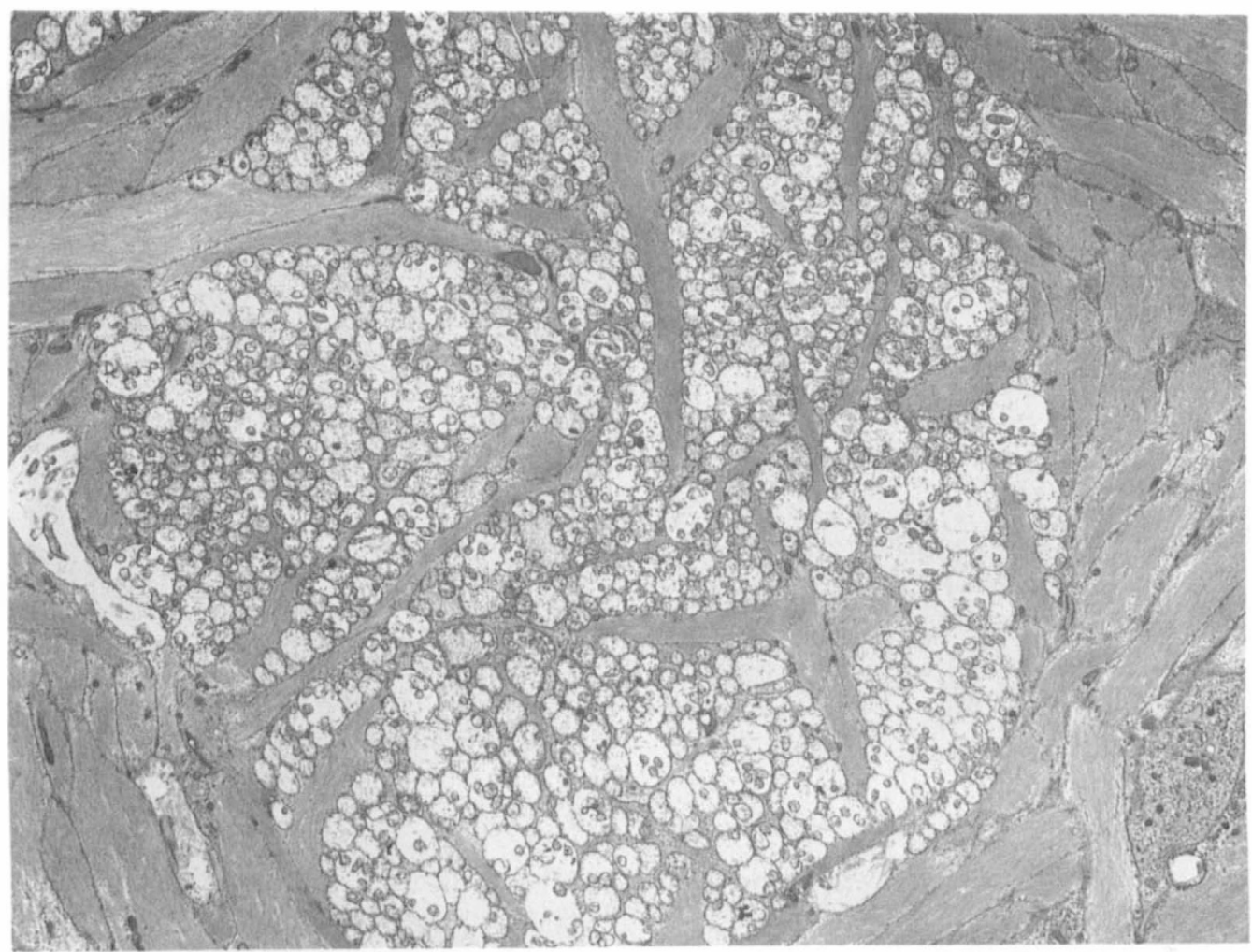

Fig. 10. Transmission electron micrograph(TEM) of the anterior (choroidal) portion of the laminacribrosa. The canal in the lamina, $30 \mu \mathrm{m}$ in diameter, is filled by axons. Trabeculae of the lamina are formed from compact sheaths of fibrous astrocyte processes which surround the canals. Other processes up to $1.5 \mu \mathrm{m}$ in diameter, extend into the canal between groups of axons. TEM $\times 3,750$.

staining material with the morphology of elastic fibres is seen within the collagenous trabeculae (Fig. 14). Where the sections are cut perpendicular to the surface of astrocyte processes, basal lamina is again seen separating the astrocytes from the surrounding collagenous tissue (Fig. 14).

\section{Discussion}

Previous studies of the anatomy of the human lamina cribrosa have employed routine histological techniques mainly to document the patterns formed by the trabeculae in the lamina and the size of the canals through which the nerve fibres pass. ${ }^{8,15}$ Transmission electron microscope studies of the lamina and the nerve fibres passing through it $^{9}$ have illustrated the intimate relationships between the axons, astrocyte processes and the fibrous trabeculae, but TEM alone gives only a limited view of the overall arrangement within the lamina. In the present study, we have used a combination of reticulin staining and immunocytochemistry to attain an integrated picture of the major elements in the lamina cribrosa which is missing from previous studies. Furthermore, we have correlated the light microscope findings with scanning EM and transmission EM data.

The integrated approach to the lamina cribrosa adopted in this study has shown several important relationships not previously described in detail. During dissection of the globes prior to preparation for transmission electron microscopy, the optic nerve and lamina separated very easily from the sclera and dural sheath suggesting that the connective tissue links between the lamina and the sclera are far from substantial. However, the most interesting new information to be derived from the present anatomical study concerns the structure of the fibrous trabeculae in the 


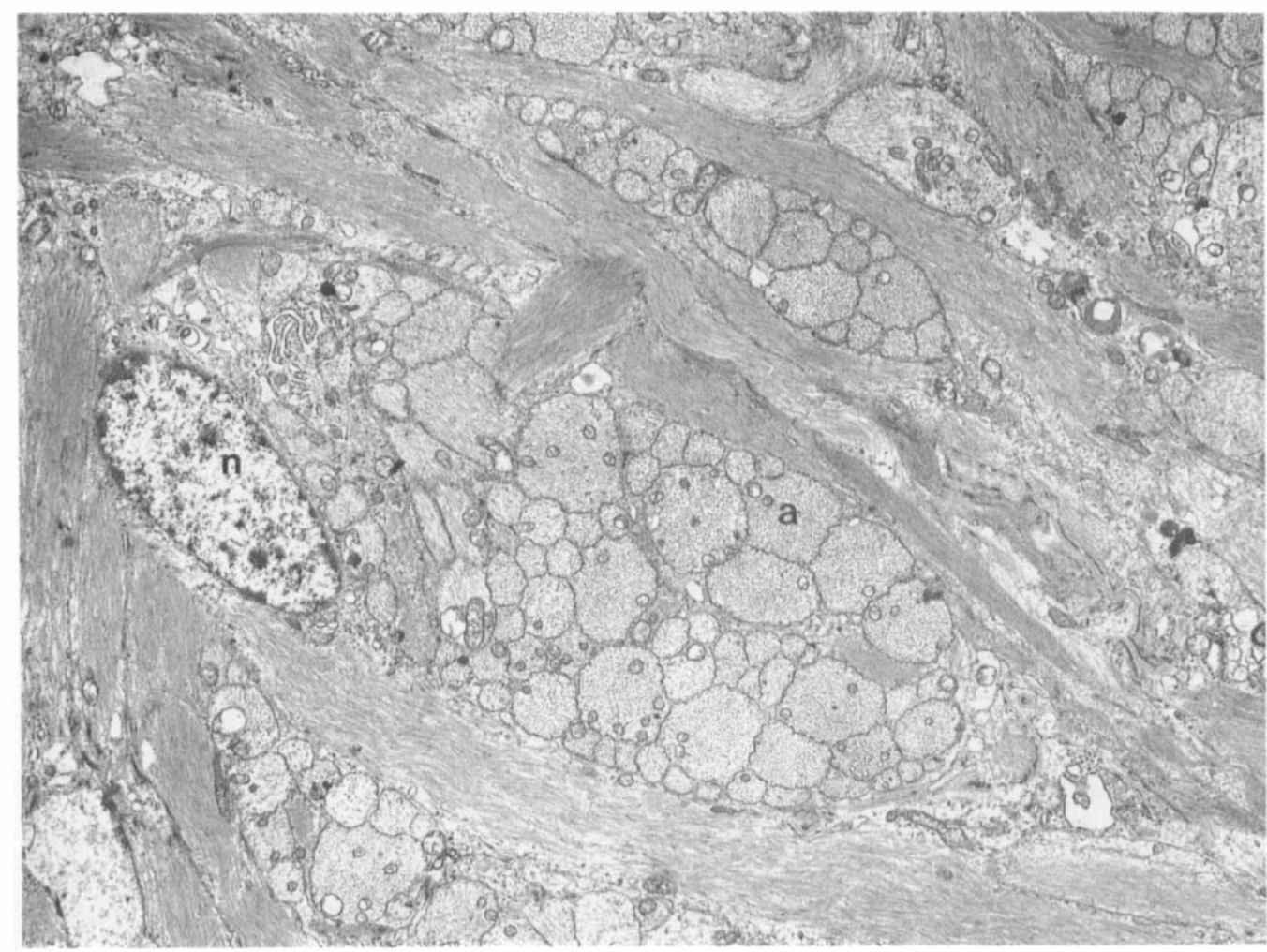

Fig. 11. Anterior portion of the lamina cribrosa showing small groups of axons separated by fibrous astrocyte processes. An astrocyte nucleus $(n)$ is seen to the left of the larger group of axons $(a)$. TEM $\times 4,500$.

lamina and the relationships of the astrocytes to the fibrous trabeculae and to the axons passing through the lamina. It was clearly seen that the fibrous trabeculae are formed from interweaving skeins of collagen fibres many of which are arranged tangentially to the canals in the lamina. It is known from previous immunocytochemical studies ${ }^{16,17}$ that the trabeculae contain collagen type III and relatively little collagen type I. However, the physical properties of the fibrous part of the lamina cribrosa are still not known. It is unclear whether the tangential arrangement of the collagen bundles around the canals in the lamina would prevent or increase deformation of the canals during alterations in intraocular pressure. ${ }^{3}$

A wide variation in the diameter of the canals in the fibrous part of the lamina cribrosa was noted in the present study but a detailed distribution of canals of different sizes in relation to their position in the lamina cribrosa was outside the scope of this investiga- tion. It has previously been observed that the canals are larger in the superior and inferior quadrants of the lamina cribrosa than in the nasal and temporal quadrant. ${ }^{15}$ From our own observations, it appears that there is considerable variation in the size of the canals and those at the lower end of the range are seen in all parts of the lamina, even in areas where the majority of canals are large. Detailed quantitative assessments of the variation in canal size are still required. Furthermore, there are few data on the changes in the structure of the lamina with age. The present study, in which eyes from patients between 18 and 56 years of age were examined, showed no qualitative differences in the structure of the lamina related to age.

The observations on immunocytochemical preparations in the present study have produced a clear picture of the web of astrocyte processes which intimately surrounds bundles of axons in the lamina cribrosa. Not only has this demonstrated the nature of the apertures 


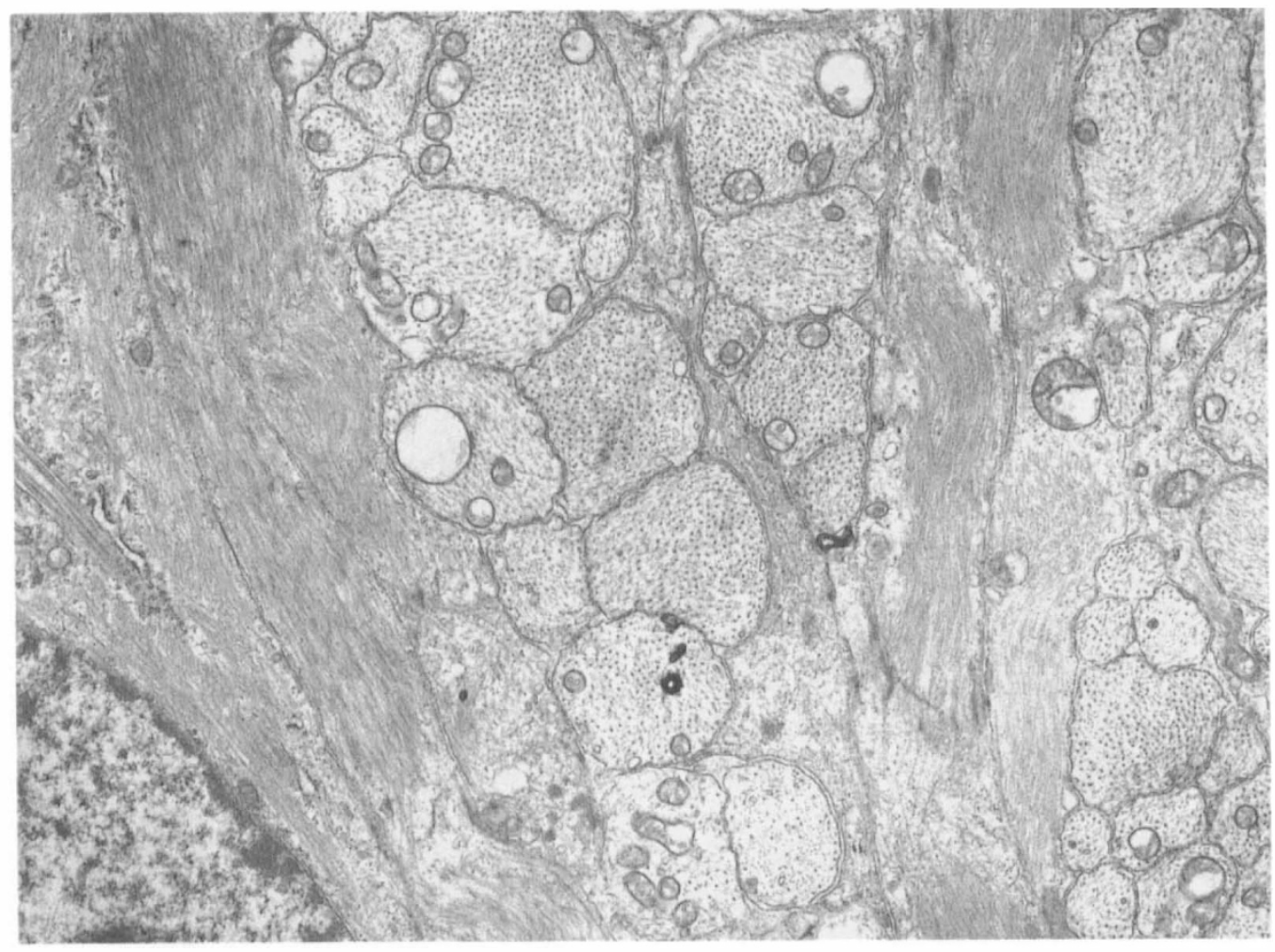

Fig. 12. Higher magnification of the same region as Figure 11 showing axons ranging from $0.5 \mu \mathrm{m}$ to $2.0 \mu \mathrm{m}$ in diameter. The larger astrocyte processes contain many intermediate filaments and show junctional cormplexes. Thin processes containing few fibrils insinuate themselves between small groups of axons. TEM $\times 10,500$.

through which the axons pass but also it has emphasised the close association of the astrocyte processes with the surfaces and cores of the fibrous trabeculae. Electron microscopy confirms the relationship previously described by Anderson ${ }^{9}$ whereby astrocyte processes surround bundles of axons. Most of the processes are rich in glial fibrillary acidic protein intermediate filaments which presumably give the processes tensile strength. However, the fine processes separating the smaller bundles of axons do not contain significant numbers of glial filaments. The physical characteristics of the astrocyte meshwork are not known and whether the astrocyte meshwork protects or damages axons under conditions of raised intraocular pressure is unclear. The smaller fibril-free processes between the axons may be concerned with metabolic support of the axons and control of the extracellular space as suggested for these cells in other parts of the brain. ${ }^{14}$
The present qualitative study of the lamina cribrosa by light and electron microscopy included scanning electron microscopy of the lamina in longitudinal and in transverse planes. As is characteristic of scanning electron microscopy, it often produces a 3-dimensional picture which emphasises the relationships between different parts of the tissue in a way that can only be suspected by light microscopy. The way in which the optic nerve enlarges posterior to the lamina cribrosa is well shown by this technique in longitudinal cuts of the optic nerve head. Similarly, the relationship of the subarachnoid space is well seen. In transverse section, the variation in diameter of the canals within the lamina cribrosa throughout the cross-section of the nerve is well demonstrated. The functional significance of this variation is unclear. Previous scanning electron microscope studies of the optic nerve have concentrated on trypsindigested preparations. ${ }^{7}$ Such enzymic diges- 


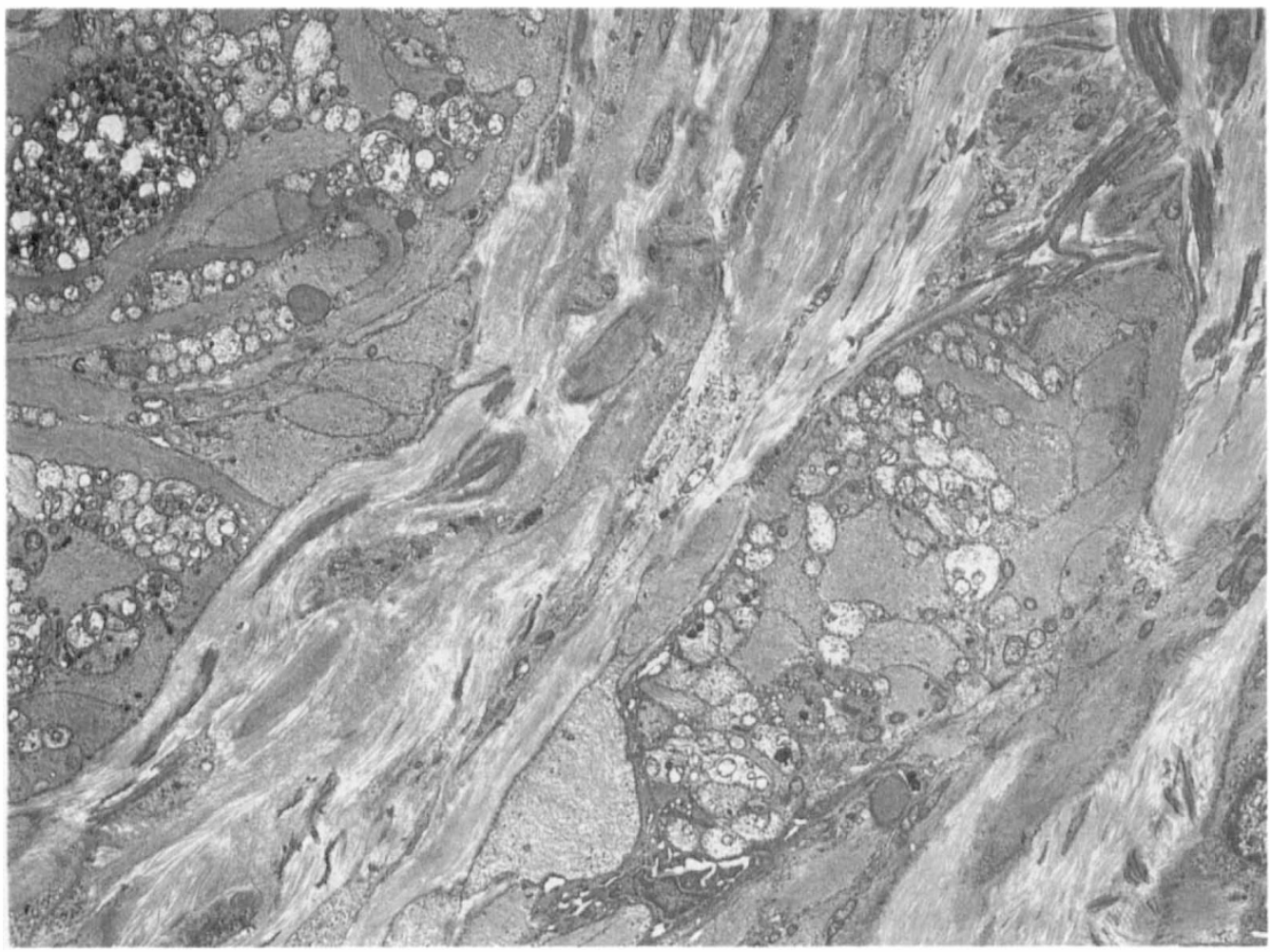

Fig.13. Posterior (scleral) part of the lamina cribrosa. A collagenous trabecula containing embedded astrocyte processes runs obliquely across the figure. Axons within a canal of the lamina cribrosa are surrounded by astrocyte processes. One axon has the morphology of an axon balloon (top left). Basement membrane surrounds astrocyte processes as they abut on to fibrous tissue. TEM $\times 3,750$.

tion by trypsin is unpredictable $\mathrm{e}^{18}$ as it is uncertain how much of the lamina itself is destroyed through enzyme digestion. None of the scanning electron microscope studies, ${ }^{7,19}$ including the present study, has entailed accurate quantitation of the size variation of the canals within the lamina cribrosa.

Although in the present study histological, immunocytochemical and ultrastructural techniques have clarified some of the intimate relationships of the various components of the lamina cribrosa, there are many unresolved questions regarding the function of the normal lamina and regarding its patterns of deformation with raised intraocular pressure. The observation that the optic disc is cupped in glaucoma has focussed attention on the lamina cribrosa as a site at which the optic nerve fibres are damaged in this disorder. ${ }^{3}$ Selective field loss in glaucoma has been correlated with a selective initial decrease in the number of nerve fibres in the superior and inferior quadrants of the optic nerve at the lamina cribrosa; ${ }^{3,19}$ axons in the nasal and temporal portions of the nerve at this point are relatively well preserved in the early stages of glaucoma. It has thus been proposed that the optic nerve damage in glaucoma may be associated with structural features of the lamina cribrosa and correlations have been made between the severity of axonal damage and the size of the canals in the collagenous (scleral) lamina cribrosa. ${ }^{3}$ As the canals in the lamina are larger in the superior and inferior quadrants of the nerve than in the nasal and temporal quadrants, it has been suggested that lack of support from the collagenous trabeculae makes the axons more vulnerable to mechanical damage in those regions. ${ }^{19}$

The results of this study indicate that the astrocyte web in the lamina cribrosa plays an important role in the mechanical and meta- 


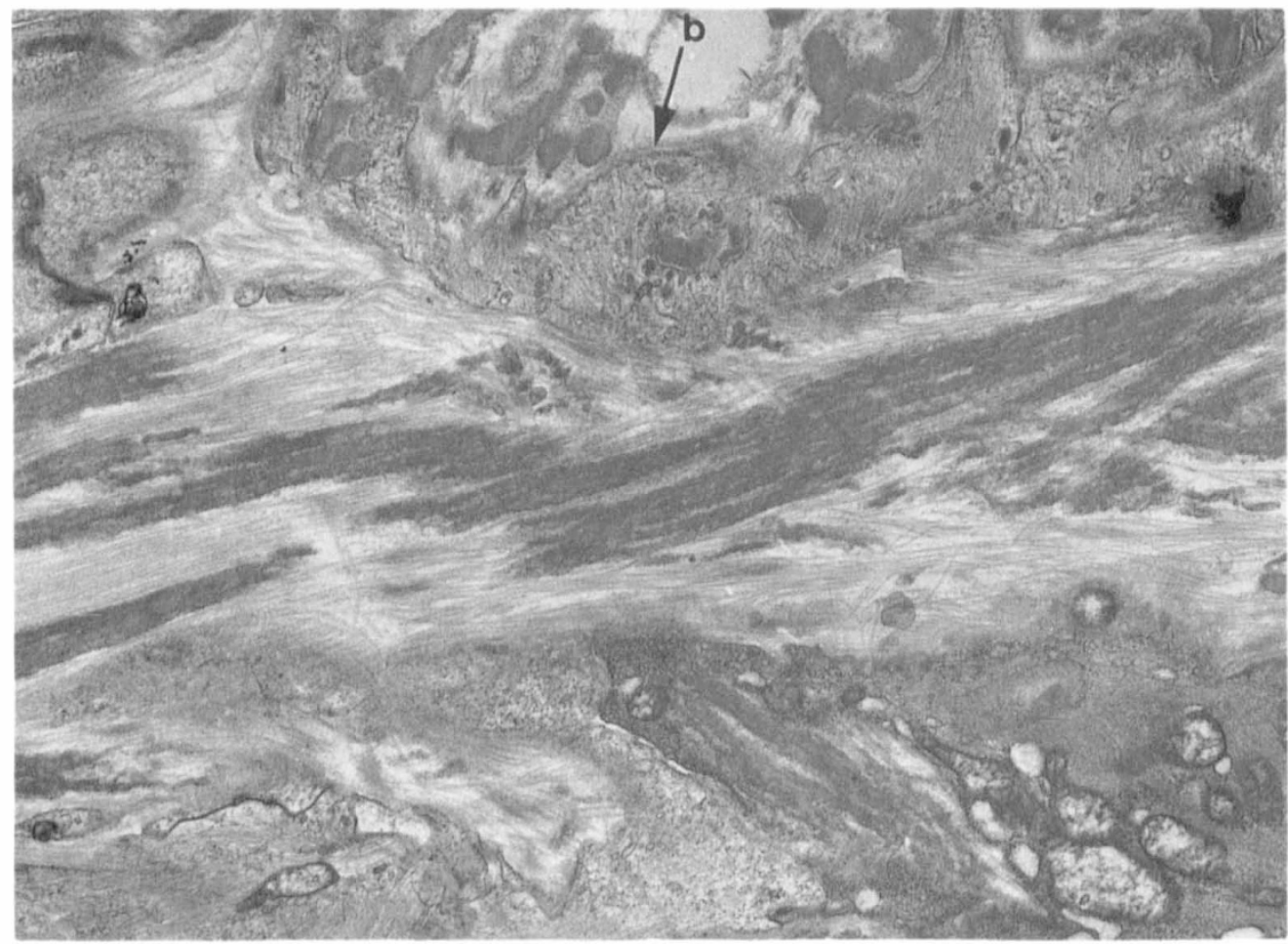

Fig. 14. Scleral Portion of the lamina cribrosa showing densely stained elastic fibres up to $0.4 \mu \mathrm{m}$ in diameter within a collagenous trabecula. Astrocyte basement membrane (b). TEM $\times 10,500$.

bolic support of optic nerve axons. Furthermore, any assessment of the changes in the lamina cribrosa in glaucoma should take into account the intimate relationships between axons, astrocytes and fibrous trabeculae as well as the complex arrangement of collagen fibres within the trabeculae.

It is a pleasure to thank Mr J. I. McGill, Professor D. Easty and Dr S. Moss who provided material used in this study. We are also grateful to Margaret Harris for typing the manuscript.

\section{References}

${ }^{1}$ Vrabec F: Glaucomatous cupping of the human optic disc. Albrecht v. Graefes Arch Klin Exp Ophthalmol 1976, 118: 223-4.

${ }^{2}$ Quigley HA, Addicks EM, Green WR, Maumenee AE: Optic nerve damage in human glaucoma. II. The site of injury and susceptibility to damage. Arch Ophthalmol 1981, 99: 635-49.

${ }^{3}$ Quigley HA: Reappraisal of the mechanisms of glaucomatous optic nerve damage. Eye 1987, 1: 318-22.

${ }^{4}$ Tso MOM and Fine BS: Electron microscopic study of human papilledema. Am J Ophthalmol 1976, 82: 424-34.
${ }^{5}$ Tso MOM and Hayreh SS: Optic disc edema in raised intracranial pressure. III. A pathologic study of experimental papilledema. Am J Ophthalmol 1977, 95: 1448-57.

${ }^{6}$ Hayreh SS: Blood supply of the optic nerve head and its role in optic atrophy, glaucoma, and oedema of the optic disc. Br J Ophthalmol 1969, 53: 721-48.

${ }^{7}$ Quigley HA and Addicks EM: Regional differences in the structure of the lamina cribrosa and their relation to glaucomatous optic nerve damage. Arch Ophthalmol 1981, 99: 137-43.

${ }^{8}$ Radius RL, Gonzales M: Anatomy of the lamina cribrosa in human eyes. Arch Ophthalmol 1981, 99: 2159-62.

${ }^{9}$ Anderson DR: Ultrastructure of human and monkey lamina cribrosa and optic nerve head. Arch Ophthalmol 1969, 82: 800-14.

${ }^{10} \mathrm{Hsu}$ SM and Raine L: Protein A, avidin and biotin in immunohistochemistry. J Histochem Cytochem 1981, 29: 1349-53.

${ }^{11}$ Palfreyman JW, Thomas DGT, Ratcliffe JG, Graham DI: Glial fibrillary acidic protein (GFAP). Purification from human fibrillary astrocytoma, development and validation of a radioimmunoassay for GFAP-like immunoreactivity. $J$ Neurol Sci 1979, 41: 101-13.

${ }^{12}$ Ponder BA and Wilkinson MM: Inhibition of endogenous tissue alkaline phosphatase with use of 
alkaline phosphatase conjugates in immunocytochemistry. J Histochem Cytochem 1981, 29: 981-4.

${ }^{13}$ Nagele RG, Doane KJ, Lee H, Wilson FJ, Roisen FJ: A method for exposing the internal anatomy of small and delicate tissues for correlated SEM/ TEM studies using polyethylene glycol embedding. J Microsc 1984, 133: 177-183.

${ }^{14}$ Peters A, Palay S, Webster HdeF: The fine structure of the nervous system: the neurons and supporting cells. Philadelphia: Saunders 1976.

${ }^{15}$ Quigley HA and Addicks EM: Regional differences in the structure of the lamina cribrosa and their relationship to glaucomatous optic nerve damage. Arch Ophthalmol 1981, 99: 137-43.
${ }^{16}$ Hernandez MR, Igoe F, Neufeld AH: Extracellular matrix of the human optic nerve head. Am JOphthalmol 1986, 102: 139-48.

${ }^{17}$ Hernandez MR, Luo XX, Igoe F, Neufeld AH: Extracellular matrix of the human lamina cribrosa. Am J Ophthalmol 1987, 104: 567-76.

${ }^{18}$ Wilkinson JM: Fragmentation of polypeptides by enzymic methods. In Darbre A ed. Practical Protein Chemistry-A handbook. New York: J Wiley 1986, 132.

${ }^{19}$ Quigley AH, Hohman RM, Addicks EM, Massof RW, Green WR: Morphologic changes in thelamina cribrosa correlated with neural loss in open-angle glaucoma. Am J Ophthalmol 1983, 95: 673-91. 